\title{
Prior dual antiplatelet therapy and thrombolysis in acute stroke
}

\section{Dual antiplatelets and thrombolysis}

Valerian L Altersberger, $\mathrm{MD}^{1 *}$, Rolf Sturzenegger, $\mathrm{MD}^{2 *}$, Silja Räty, $\mathrm{MD}^{3}$, Christian Hametner, $\mathrm{MD}^{4}$, Jan F Scheitz, $\mathrm{MD}^{5}$, Solène Moulin, $\mathrm{MD}, \mathrm{PhD}^{6}$, Sophie A van den Berg, $\mathrm{MD}^{7}$, Andrea Zini, $\mathrm{MD}^{8}$, Stefania Nannoni, $\mathrm{MD}^{9}$, Mirjam R Heldner, $\mathrm{MD}^{10}$, Dejana R Jovanovic, MD, $\mathrm{PhD}^{11,12}$, Nicolas Martinez-Majander, $\mathrm{MD}^{3}$, Marjaana Tiainen, $\mathrm{MD}, \mathrm{PhD}^{3}$, Kati Valkonen, $\mathrm{MD}^{3}$, Anne Berberich, $\mathrm{MD}^{4}$, Hebun Erdur, $\mathrm{MD}^{5}$, Charlotte Cordonnier, $\mathrm{MD}, \mathrm{PhD}^{13}$, Nils Peters, $\mathrm{MD}^{1,20}$, Kiran M Gopisingh ${ }^{7}$, Guido Bigliardi, $\mathrm{MD}^{14}$, Davide Strambo, $\mathrm{MD}^{9}$, Gian Marco De Marchis, MD, MSc ${ }^{1}$, George Ntaios, MD, MSc, $\mathrm{PhD}^{15}$, Carlo W Cereda, $\mathrm{MD}^{16}$, Susanne Wegener, $\mathrm{MD}^{17}$, Georg Kägi, $\mathrm{MD}^{18}$, Alessandro Pezzini, $\mathrm{MD}^{19}$, Visnja Padjen, MD, $\mathrm{PhD}^{11}$, Marcel Arnold, $\mathrm{MD}^{10}$, Patrik Michel, $\mathrm{MD}^{9}$, Laura Vandelli, $\mathrm{MD}^{14}$, Paul J Nederkoorn, $\mathrm{MD}, \mathrm{PhD}^{7}$, Didier Leys, $\mathrm{MD}, \mathrm{PhD}^{13}$ Christian $\mathrm{H}_{\text {Nolte, }} \mathrm{MD}^{5}$, Peter A Ringleb, $\mathrm{MD}^{4}$, Sami Curtze, $\mathrm{MD}, \mathrm{PhD}^{3}$, Stefan $\mathrm{T}$ Engelter, $\mathrm{MD}^{1,20 *}$, Henrik Gensicke, $\mathrm{MD}^{1,20 *}$, for the Thrombolysis in Stroke Patients (TRISP) collaborators

* contributed equally

1. Stroke Center and Department of Neurology, University Hospital Basel and University of Basel, Switzerland

2. Department of Neurology, Kantonsspital Graubuenden, Chur, Switzerland

3. Department of Neurology, University of Helsinki and Helsinki University Hospital, Finland

4. Department of Neurology, University Hospital Heidelberg, Germany

5. Department of Neurology and Center for Stroke Research Berlin, Charité, Berlin, Germany

6. Department of Neurology, Centre Hospitalier Universitaire de Reims, France

7. Department of Neurology, Amsterdam University Medical Center, University of Amsterdam, the Netherlands

8. IRCCS Istituto di Scienze Neurologiche di Bologna, Department of Neurology and Stroke Center, Maggiore Hospital, Bologna, Italy

9. Department of Neurology, Centre Hospitalier Universitaire Vaudois and University of Lausanne, Switzerland

This article has been accepted for publication and undergone full peer review but has not been through the copyediting, typesetting, pagination and proofreading process which may lead to differences between this version and the Version of Record. Please cite this article as doi: 10.1002/ana. 25850 
10. Department of Neurology, Inselspital, Bern University Hospital and University of Bern, Switzerland

11. Neurology Clinic, Clinical Centre of Serbia, Serbia (VP, DRJ)

12. Medical Faculty, University of Belgrade, Belgrade, Serbia (DRJ)

13. Univ. Lille, Inserm, CHU Lille, U1172 - LilNCog - Lille Neuroscience \& Cognition, F59000 Lille, France

14. Stroke Unit, Neurology Clinic, Department of Neuroscience, Ospedale Civile "S. Agostino-Estense", Modena University Hospital, Modena, Italy

15. Department of Internal Medicine, Faculty of Medicine, School of Health Sciences, University of Thessaly, Larissa, Greece

16. Stroke Center and department of Neurology, Neurocenter of Southern Switzerland, Lugano, Switzerland

17. Department of Neurology, University Hospital Zurich and University of Zurich, Switzerland

18. Department of Neurology, Kantonsspital St. Gallen, Switzerland

19. Department of Clinical and Experimental Sciences, Neurology Clinic, University of Brescia, Italy

20. Neurorehabilitation, University of Basel and University Department of Geriatic Medicine FELIX PLATTER, University of Basel, Switzerland.

\section{Correspondence to:}

Dr. Henrik Gensicke

Department of Neurology

University Hospital Basel

Petersgraben 4

CH - 4031 Basel

Phone: +41613286727

Fax: +41612654452

E-mail: henrik.gensicke@usb.ch 


\section{Word count:}

Title (characteristics): 56

Running head (characteristics): 32

Letter to the editor: 451

References: 5

Tables: 1

\section{Letter to the editor}

The available evidence on safety of intravenous thrombolysis (IVT) in acute ischemic stroke patients (AIS) on dual antiplatelet therapy (DAPT) is contradictory ${ }^{1-4}$. In particular, clinicians are uncertain whether to use or refrain from IVT in high risk patients, like those with pre-stroke disability who receive DAPT.

Using data from the prospective Safe Implementation of Treatments in Stroke International Stroke Thrombolysis Register (SITS-ISTR), Tsivgoulis et al. recently showed that AIS patients on DAPT had similar rates of symptomatic intracerebral hemorrhage (sICH), poor functional outcome and mortality after IVT compared to AIS patients without antiplatelet therapy (APT). ${ }^{3}$ We think that this analysis, despite the use of propensity score matching, has some limitations. First, the analysis excluded patients with pre-stroke disability (pre-stroke modified Rankin Scale 2-5), subjects who are more likely to be on DAPT ${ }^{5}$; second, the dataset had a relatively high percentage of missing data (10.5\% missing data on APT status, $27 \%$ missing data on 3month outcome and $7.4 \%$ on follow-up neuroimaging).

Aiming to overcome these limitations and to increase evidence about safety of DAPT in IVT, we analysed data from the prospective international Thrombolysis in Ischemic Stroke Patients (TRISP) registry. All AIS patients - independently of pre-stroke disability - were included. Primary outcomes included sICH (ECASS-II-criteria), poor functional outcome (defined as modified Rankin Scale 3-6) and mortality (both at three months). DAPT was defined as any combination of antiplatelet therapy with aspirin, clopidogrel, ticagrelor, prasugrel or dipyridamole. Patients without APT and without anticoagulants (AC) served as the comparison group. We performed logistic regression analyses and calculated odds ratios with 95\% 
confidence intervals [OR, 95\%-CI] with adjustment for potentially outcome-modifying variables.

Among 15’156 IVT-treated stroke patients, 8’433 (55.6\%) had no APT and no AC, 5’611 (37.0\%) were on single APT, 404 (2.7\%) were on DAPT and 708 (4.7\%) patients were on AC. Data completeness rate was $>93 \%$ (missing data on APT: $1.1 \%$ [n=182]; 3-month outcome: 3.8\% [613]; sICH: 1.4\% [218]). The proportion of patients with relevant pre-stroke disability (pre-mRS 3-5) in the DAPT group (10.6\%) was significantly higher in comparison to patients without APT (6.0\%). Besides, patients on DAPT were older and more often had cardiovascular risk factors than patients without APT. The risk of sICH (ORadjusted 1.29[0.82-2.05]), poor outcome (ORadjusted 0.69[0.34-1.20]), and death (ORadjusted 1.48[0.87-2.54]) did not differ significantly between IVT-treated patients on DAPT and no APT (Table 1).

In line with Tsivgoulis et al., our study strongly supports the evidence of not withholding IVT in AIS-patients on DAPT using a different, large prospective, multicenter and multinational data set. Strengths of our study are the high data completeness and - as a refinement - the clarification that safety of IVT in AIS-patients on DAPT applies also to patients with relevant pre-stroke disability.

\section{Author contribution:}

VLA designed/conceptualized the study, collected data, analyzed/interpreted the data, drafted the manuscript.

RS designed/conceptualized the study, revised the manuscript.

STE and HG designed/conceptualized and initiated the study, supervised the study, collected data, analyzed/interpreted the data, revised the manuscript.

All other authors collected data, analyzed/interpreted the data and revised the manuscript.

\section{Potential Conflicts of Interest}

Valerian L. Altersberger, Rolf Sturzenegger, Gian Marco De Marchis, Christian Hametner, Anne Berberich, Jan F Scheitz, Sophie A. van den Berg, Stefania Nannoni, Alessandro Pezzini, 
Guido Bigliardi, Laura Vandelli, Silja Räty, Kati Valkonen, Nicolas Martinez-Majander, Marjaana Tiainen, Patrik Michel, Kiran M Gopisingh, Davide Strambo, Paul J. Nederkoorn, Mirjam R. Heldner, Solène Moulin, Hebun Erdur, Henrik Gensicke, George Ntaios and Sami Curtze report no relevant disclosures.

Carlo W. Cereda has received modest honoraria for scientific advisory board from BoehringerIngelheim (producer of alteplase).

Charlotte Cordonnier received speaker honoraria from Boehringer-Ingelheim (producer of alteplase). She is a member of DSMB (unpaid) of ATTEST-2 and FivHEMA trials in which alteplase is studied.

Susanne Wegener received research funds by Boehringer- Ingelheim (producer of alteplase).

Didier Leys reports participation in trials sponsored by Boehringer-Ingelheim (producer of alteplase).

Christian H. Nolte has received modest speaker honoraria from Boehringer Ingelheim (producer of alteplase).

Andrea Zini has received funding for speaker honoraria and consulting fees as well as for scientific advisory board from Boehringer-Ingelheim (producer of alteplase).

Dejana R. Jovanovic: travel or speaker honoraria from Boehringer Ingelheim (producer of alteplase) and is member of scientific advisory board for Boehringer Ingelheim.

Visnja Padjen: travel or speaker honoraria from Boehringer Ingelheim (producer of alteplase).

Georg Kägi has received modest honoraria for travel and advisory board from BoehringerIngelheim (producer of alteplase).

Nils Peters has received funding for travel and served on scientific advisory boards for Boehringer-Ingelheim. (producer of alteplase).

Peter A. Ringleb has received modest honoraria for lectures and advisory board from Boehringer-Ingelheim (producer of alteplase). The University Hospital Heidelberg is sponsor of the ECASS4-trial, examining the role of rtPA in an extended time-window, which is financed by Boehringer-Ingelheim.

Marcel Arnold received Speaker honoraria from Bayer, Boehringer Ingelheim; Scientific advisory board honoraria from Boehringer Ingelheim. 
Stefan T. Engelter has received funding for travel or speaker honoraria from Bayer BoehringerIngelheim (producer of alteplase). He has also served on scientific advisory boards for Boehringer-Ingelheim.

\section{References}

1. Tsivgoulis G, Katsanos AH, Mavridis D, et al. Intravenous Thrombolysis for Ischemic Stroke Patients on Dual Antiplatelets. Ann Neurol. 2018 Jul;84(1):89-97.

2. Robinson TG, Wang X, Arima H, et al. Low- Versus Standard-Dose Alteplase in Patients on Prior Antiplatelet Therapy: The ENCHANTED Trial (Enhanced Control of Hypertension and Thrombolysis Stroke Study). Stroke. 2017 Jul;48(7):1877-1883.

3. Powers WJ, Rabinstein AA, Ackerson T, et al. Guidelines for the Early Management of Patients With Acute Ischemic Stroke: 2019 Update to the 2018 Guidelines for the Early Management of Acute Ischemic Stroke: A Guideline for Healthcare Professionals From the American Heart Association/American Stroke Association. Stroke. 2019 Dec;50(12):e344-e418.

4. Malhotra K, Katsanos AH, Goyal N, et al. Safety and efficacy of dual antiplatelet pretreatment in patients with ischemic stroke treated with IV thrombolysis: A systematic review and meta-analysis. Neurology. 2020 Feb 18;94(7):e657-e666.

5. Gensicke H, Strbian D, Zinkstok SM, et al. Intravenous Thrombolysis in Patients Dependent on the Daily Help of Others Before Stroke. Stroke. 2016 Feb;47(2):450-6. 
$\underline{\text { Table 1. Multivariable analysis of outcomes (odds adjusted for variables with } \mathbf{p}<0.1 \text { in the univariable analysis). Odds ratio (95\% }}$

\section{confidence interval), p-value.}

\begin{tabular}{|c|c|c|c|}
\hline & \multicolumn{3}{|c|}{ Outcome measures } \\
\hline Putative predicting variables & sICH & Poor outcome & Mortality \\
\hline Dual antiplatelet vs no APT and no AC & $1.29(0.82-2.05)^{1} \mathrm{p}=0.272$ & $0.69(0.34-1.20)^{2} \mathrm{p}=0.185$ & $1.48(0.87-2.54)^{3} p=0.150$ \\
\hline
\end{tabular}

1: adjusted for age, NIHSS, dual antiplatelet

2: adjusted for age, sex, stroke to needle time, NIHSS on admission, relevant pre-stroke disability, atrial fibrillation, diabetes, hypertension, hypercholerserolemia, smoking, coronary artery disease, prior ischemic stroke, RR systolic on admission, glucose on admission, creatinine on admission, dual antiplatelet

3: adjusted for age, NIHSS on admission, relevant pre-stroke disability, atrial fibrillation, glucose on admission, creatinine on admission, dual antiplatelet

Abbreviations:

sICH: symptomatic intracerebral hemorrhage

APT: antiplatelet therapy

DAPT: dual antiplatelet therapy

AC: anticoagulation

NIHSS: National Institutes of Health Stroke Scale 